\title{
FPU Recurrence in the Coupled Blood Pressure Dynamics in Magisterial and Small Arteries Networks and the Character of its Interaction with the Heart Electrical Activity
}

Shmid AV, Novopashin MA and Berezin AA*

EC-leasing, Varshavskoye Shosse, Moscow, Russia

\begin{abstract}
Computer model simulating the coupled blood pressure dynamics in magisterial and small arteries with the heart electrical dynamics has been suggested. The Fermi-Pasta-Ulam auto recurrence in the description of the electrical activity of the heart has demonstrated the universal role of the FPU recurrence in the study of dynamical systems. The heart electrical dynamics was described by the coupled Van der Pol differential equations with time lags, linked with two additively coupled nonlinear differential equations circumscribing the blood pressure dynamics in the networks of magisterial and small arteries. The arterial blood pressure dynamics was interpreted as coupled FPU recurrences showing a rich variety of resulting FPU spectra, which were referred to different states of Cardio Vascular System. Synchronous registering of the real ECG and Pulse Wave Fourier dynamic images allowed to unify the characteristic Fourier pictures of the heart electrical activity with the hydrodynamic blood parameters developing in the networks of two types of arteries. The computer study of the suggested model and comparison of its results with the real data proved that the ECG Fourier parameters coupled with the Pulse Wave Fourier parameters form the FPU spectra that increase stability of Cardio Vascular System and can be used for diagnostics as well as for evaluation of the therapeutic arrangements results.
\end{abstract}

Keywords: Cardio vascular; Blood; Heart; Myocardial; Cardiac

\section{Introduction}

In our previous paper [1], it was suggested using the Fermi-PastaUlam auto recurrence phenomenon concept in the description of the electrical activity of the heart. At the same, time the character of the dynamics of blood pressure in magisterial and small arteries networks shows that it is not simply providing the blood stream propagation but plays a significant role in stabilizing the heart functioning within certain parameters as well as probably facilitates the flow of myocardial infarct. Such assumptions came from qualitatively different pictures of blood pressure profiles in magisterial and small arteries. Assume that the blood pushed by the heart beat into aorta represents a solitary wave similar to that of Yuen and Lake one obtained in their study of deep water wave properties [2]. The role of "heart" in their experimental research played a mechanical wave producer. They developed a mathematical model for description of the deep wave dynamics within the Nonlinear Shrodinger (NSE) equation and found a good agree between the mathematical solution and experimental data. Moreover, they proved forming the FPU recurrence profile during propagation of deep waves in experimental channels. In accordance with the NSE, the FPU recurrence functions were odd ones. The deep-water waves, propagation process in their experiment ended on a shallow surface. It is known, that the dynamics of shallow water waves is described by the Korteveg de Vries equation [3]. Zabusky and Kruskal showed in their mathematical model that imposing periodical boundary condition on the KdV equation forms the FPU recurrence spectrum. Considering periodical conditions as heart beatings pushing blood in the "shallow" part consisting of small arteries network and even solution of the KdV equation, one can get the FPU recurrence with even functions in its spectrum. Thus, the dynamics of blood in both networks can be given as a mixed odd-even FPU recurrence spectrum reflecting the state of big and small arteries together with the heart state dynamics.

\section{Mathematical Model}

The basic core for mathematical model was taken from the coupled
Van der Pol model of the heart electrical activity, given in the paper [4]. Trying to simplify the solutions of the NSE and KdV there were used nonlinear second order differential equations with odd non linearity for NSE and even non linearity for KdV equation.

The coupled Van der Pol equations by agreeing coefficients were additively linked to the equations substituting NSE and KdV, forming a new model looking in the following way:

$$
\begin{aligned}
& \frac{d^{2} M_{1}}{d t^{2}}-a_{1}\left(1-Y_{1}\right) \frac{d M_{1}}{d t}+\omega_{1}^{2}\left(1+\alpha_{1} M_{2}\right) M_{1}=c_{1} \frac{d^{2} M_{2}}{d t^{2}}+d_{1} F_{1}+d_{2} F_{2} \\
& b_{1} Y_{1}+T_{1} \frac{d Y_{1}}{d t}=M_{1}^{2} \\
& \frac{d^{2} H_{1}}{d t^{2}}+\frac{d H_{1}}{d t}+\left(1+e_{1} H_{1}^{2}+e_{2} H_{1}^{4}+e_{3} H_{1}^{6}\right) H_{1}=k_{1} M_{1}+k_{2} H_{2} \\
& \frac{d^{2} M_{2}}{d t^{2}}-a_{2}\left(1-Y_{2}\right) \frac{d M_{2}}{d t}+\omega_{2}{ }^{2}\left(1+\alpha_{2} M_{1}\right) M_{2}=c_{2} \frac{d^{2} M_{1}}{d t^{2}}+d_{1} F_{1}+d_{2} F_{2} \\
& b_{2} Y_{2}+T_{2} \frac{d Y_{2}}{d t}=M_{2}{ }^{2} \\
& \frac{d^{2} H_{2}}{d t^{2}}+\frac{d H_{2}}{d t}+j_{1} H_{2}{ }^{3}+j_{2} H_{2}{ }^{5}+j_{3} H_{2}{ }^{7}+H_{2}=k_{3} M_{2}+k_{4} H_{1}
\end{aligned}
$$

*Corresponding author: Andrey Berezin, EC-leasing, Varshavskoye Shosse Moscow 113405, Russian Federation, Tel: 89671280467; E-mail: artparis@mail.ru

Received December 12, 2017; Accepted December 27, 2017; Published December 30, 2017

Citation: Shmid AV, Novopashin MA, Berezin AA (2017) FPU Recurrence in the Coupled Blood Pressure Dynamics in Magisterial and Small Arteries Networks and the Character of its Interaction with the Heart Electrical Activity. Cardiovasc Pharm Open Access 6: 230. doi: 10.4172/2329-6607.1000230

Copyright: ( 2017 Shmid AV, et al. This is an open-access article distributed under the terms of the Creative Commons Attribution License, which permits unrestricted use, distribution, and reproduction in any medium, provided the original author and source are credited. 
Citation: Shmid AV, Novopashin MA, Berezin AA (2017) FPU Recurrence in the Coupled Blood Pressure Dynamics in Magisterial and Small Arteries Networks and the Character of its Interaction with the Heart Electrical Activity. Cardiovasc Pharm Open Access 6: 230. doi: 10.4172/23296607.1000230

Page 2 of 6

Where $M_{1}$ - is the magnitude proportional to the dynamic electric potential of the whole myocardium, $M_{2}$ - is the magnitude proportional to the dynamic electric potential of the myocyte locality on the surface of the myocardium, $H_{1}$ - is the magnitude proportional blood pressure in the small arterial network, $H_{2}$ - is the magnitude proportional blood pressure in the magisterial arterial network, $k_{1}, k_{2}, k_{3}, k_{4}$ - agreeing coefficients, $e_{1}, e_{2}, e_{3}, j_{1}, j_{2}, j_{3}$ -constant coefficients, $Y_{1}$ - is the magnitude proportional to the time lag which requires the propagation of the electric impulse in the myocardium, $Y_{2}$ - is the magnitude proportional to the time lag which requires the propagation of the electric impulse in the myocyte locality, $b_{1}$ - is the magnitude proportional to the square of the myocardium, $b_{2}$ - is the magnitude proportional to the square of the myocyte locality, $T_{1}$ - is the magnitude proportional to the time of the myocardium contraction, $T_{2}$ - is the magnitude proportional to the period of oscillations in the myocyte locality, $\omega_{1}, \omega_{2}$ - are the beating frequencies equal to $1, F_{1}$ - is the perturbation function of the resonant external medium effect on the heart at the frequency of about $1 \mathrm{~Hz}, F_{2}$ - is the perturbation function of the resonant

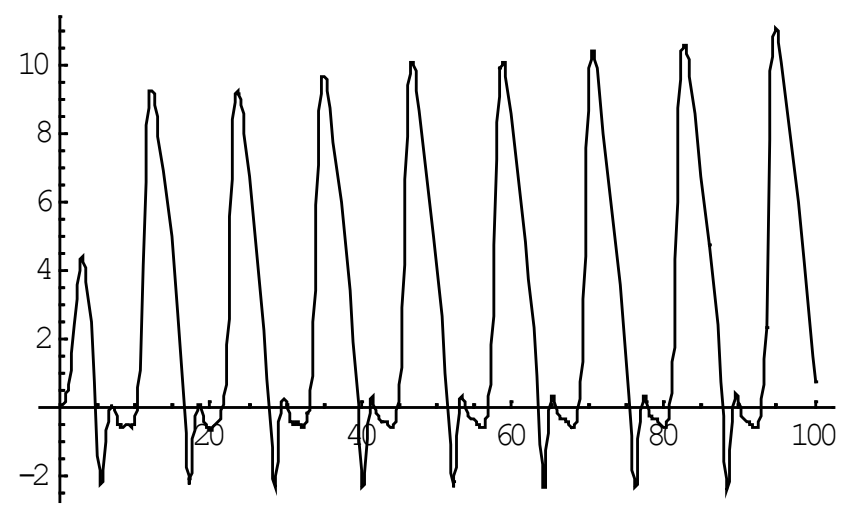

Figure 1: Model ECG of a healthy person. external medium effect on the heart at the frequency of about 20 Hz., $c_{1}, c_{2}, d_{1}, d_{2}, \alpha_{1}, \alpha_{2}$ - are the constants.

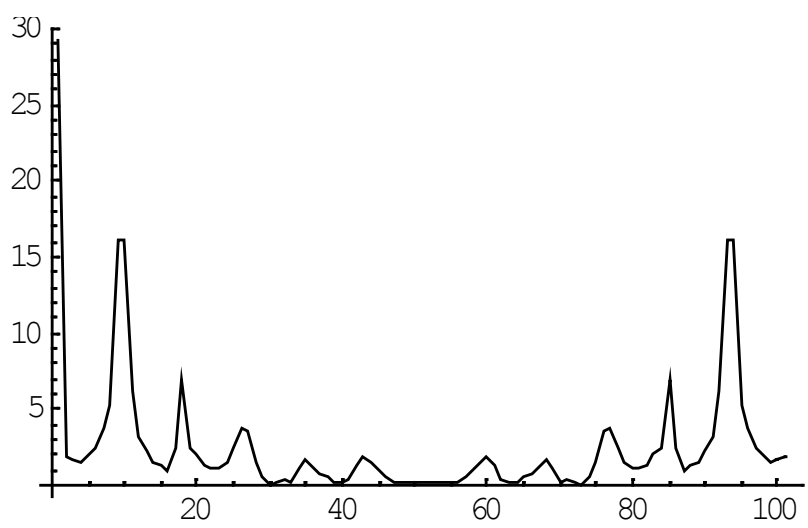

Figure 2: Fourier image of a model ECG of a healthy person.

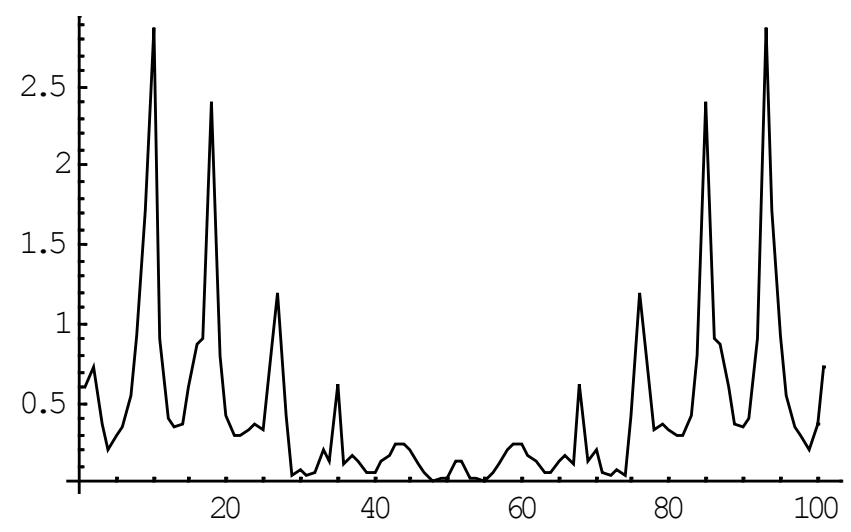

Figure 3: Fourier image of a Pulse Wave of a healthy person.

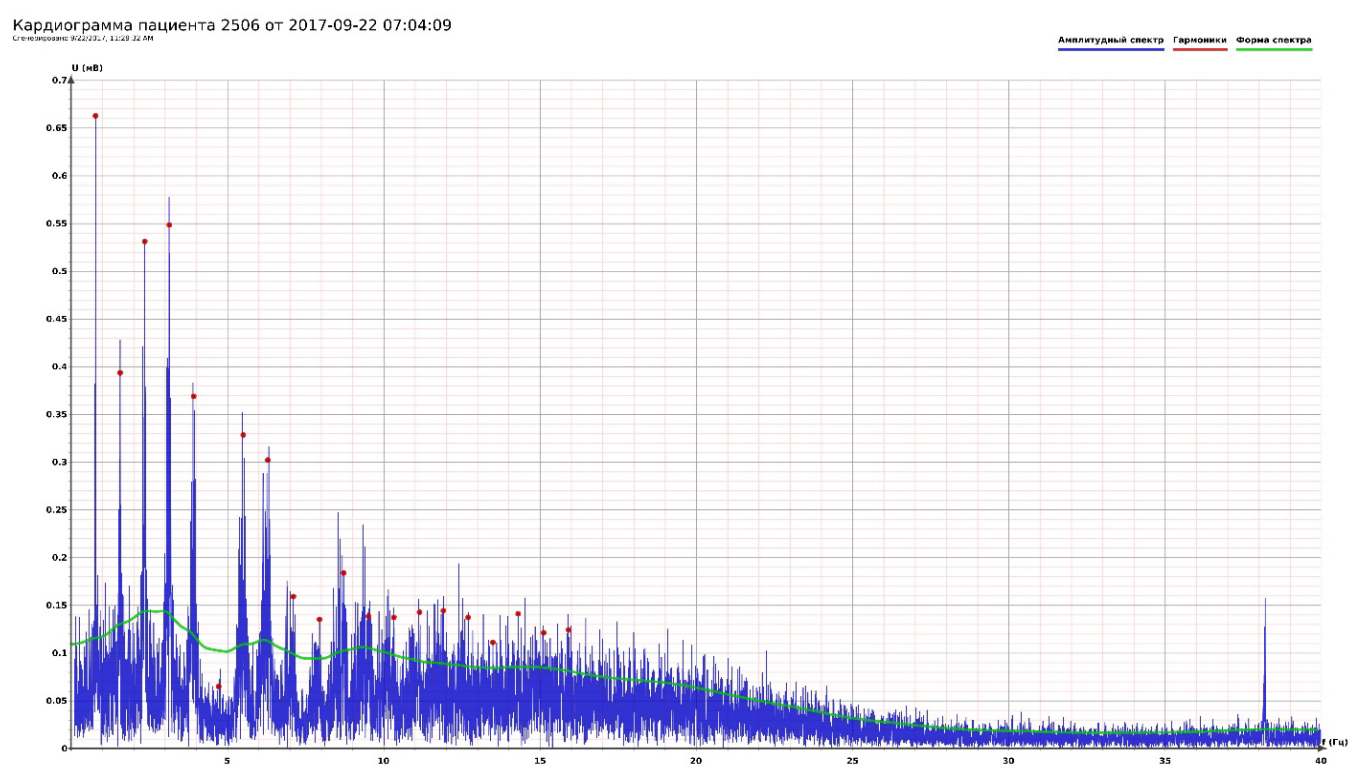

Figure 4: Real Fourier image of a healthy person ECG. 
Citation: Shmid AV, Novopashin MA, Berezin AA (2017) FPU Recurrence in the Coupled Blood Pressure Dynamics in Magisterial and Small Arteries Networks and the Character of its Interaction with the Heart Electrical Activity. Cardiovasc Pharm Open Access 6: 230. doi: 10.4172/23296607.1000230

Page 3 of 6

\section{The Study of the Model}

The computer study of the developed model allowed to get the model solutions corresponding to the healthy state of the heart and magisterial and small arteries networks coupled functioning. Figures 1-3 show model ECGs and Pulse Wave Fourier images correspondingly.

The model Fourier images were compared with the ECG and Pulse Wave Fourier data of healthy patients. As it can be seen from the graphs (Figures 4 and 5), the model Fourier pictures of a healthy patient correspond to the mathematical model solutions.
Next step of modeling was to decrease the myocardial surface by $50 \%$ as a model of myocardial infarct. Previous model [4], showed close to fibrillation pictures whereas modeling such situation within a new model (1) demonstrated only a small deterioration of the ECG picture (Figure 6 ) and its Fourier image (Figure 7) as well as the images of the magisterial and small arteries networks dynamics (Figure 8).

The model images were compared with the ECG and Pulse Wave Fourier data of a patient with a stent having cardiac problems. As it can be seen from the graphs (Figures 9 and 10), the model Fourier pictures of a patient with a stent correspond to the model

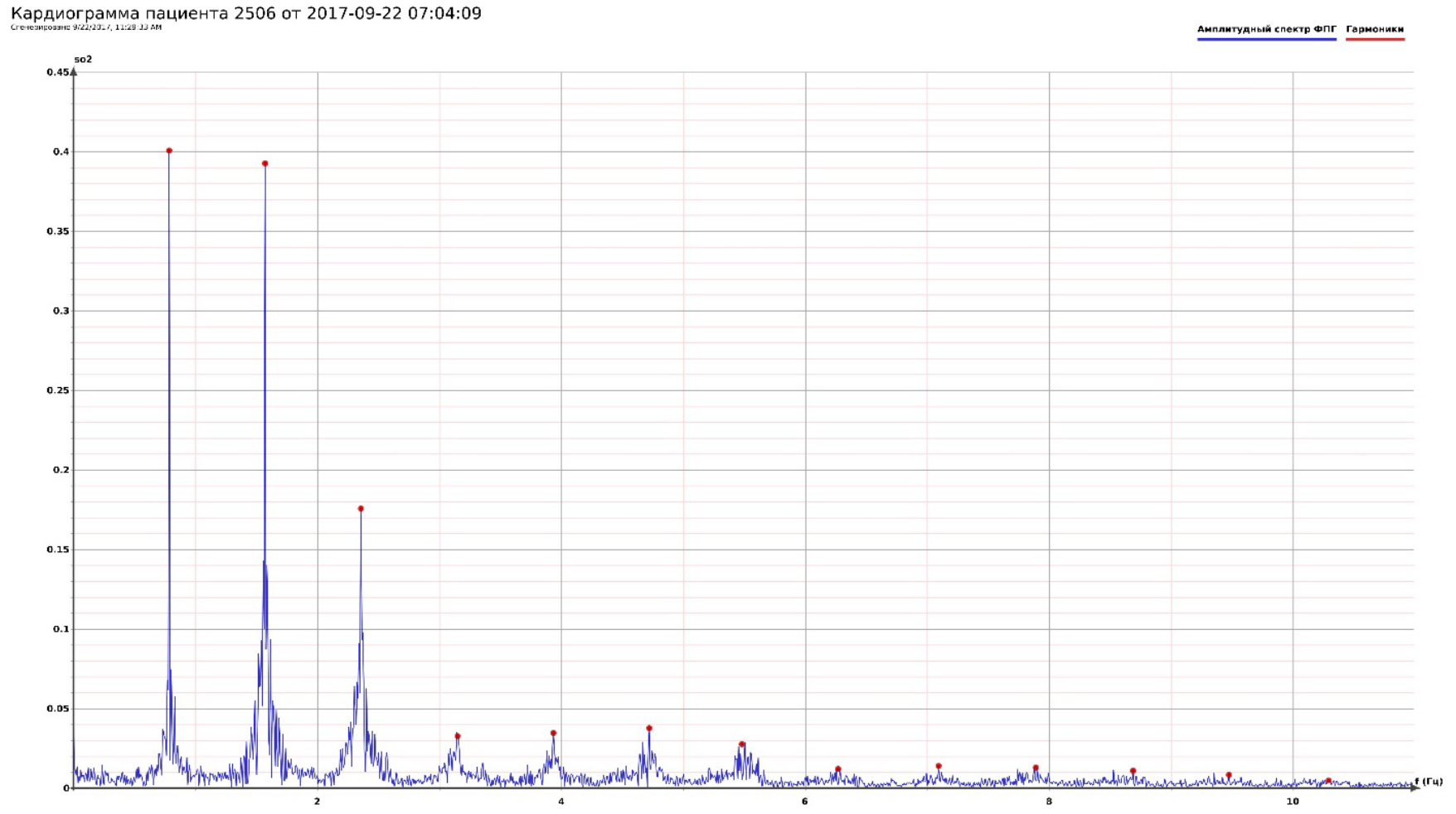

Figure 5: Real Fourier image of a healthy person Pulse Wave.

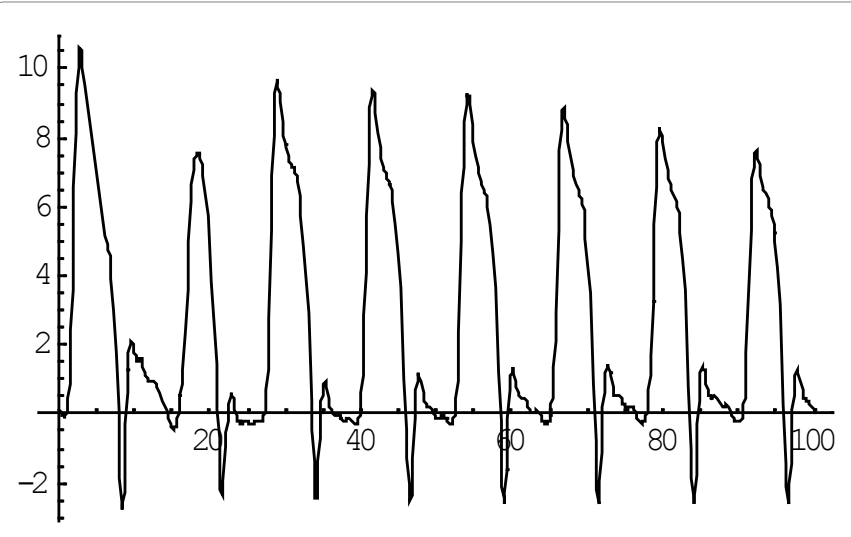

Figure 6: Model ECG with myocardial infarct.

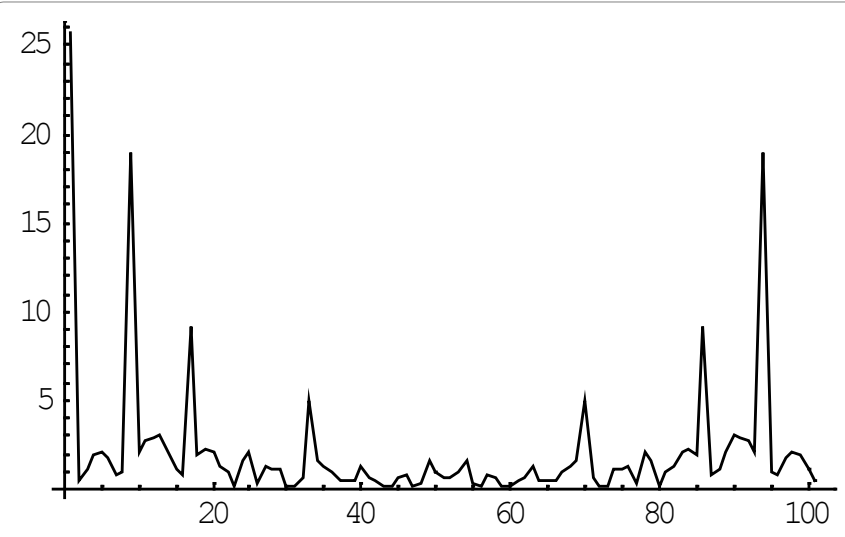

Figure 7: Fourier image of a model ECG with myocardial infarct. 
Citation: Shmid AV, Novopashin MA, Berezin AA (2017) FPU Recurrence in the Coupled Blood Pressure Dynamics in Magisterial and Small Arteries Networks and the Character of its Interaction with the Heart Electrical Activity. Cardiovasc Pharm Open Access 6: 230. doi: 10.4172/23296607.1000230

Page 4 of 6

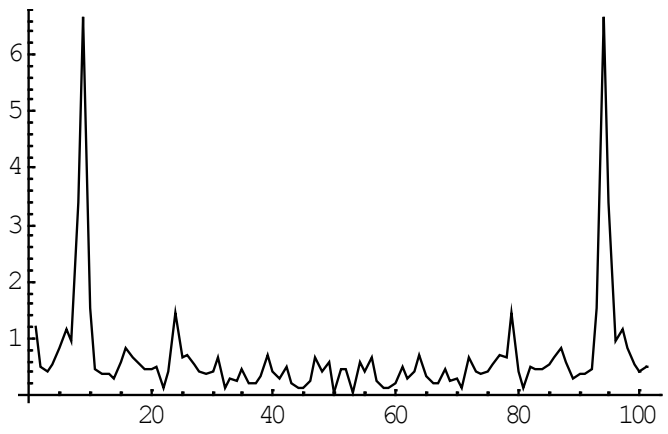

Figure 8: Fourier image of a model Pulse Wave with myocardial infarct. myocardial problem solutions (Figures 7 and 8). After a course of therapy, the ECG and PW images of the patient have improved as in can be seen in the Figures 11 and 12 .

Modeling of fibrillation within a new model proved an ability of the coupled system of the heart and magisterial and small arteries networks to withstand the cardiovascular problems happened due to different reasons. In particular, creating model fibrillation in a new model requires five times less magnitude of the amplitude of external influence to compare with the magnitude of the amplitude in the previous modeling the state of heart fibrillation [4]. The arterial networks stabilize the whole Cardiovascular functioning (Figures 13-15).

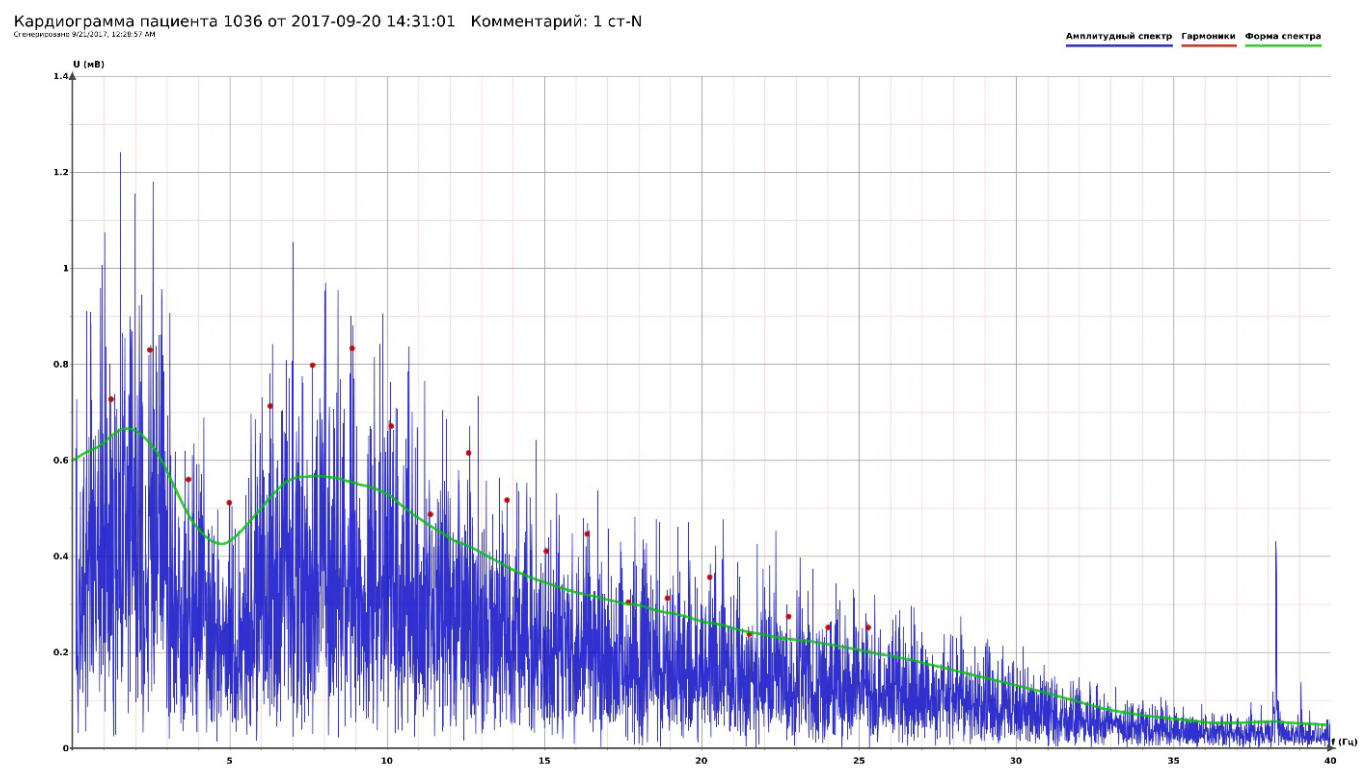

Figure 9: Real Fourier ECG image of a patient with a cardiac problem.

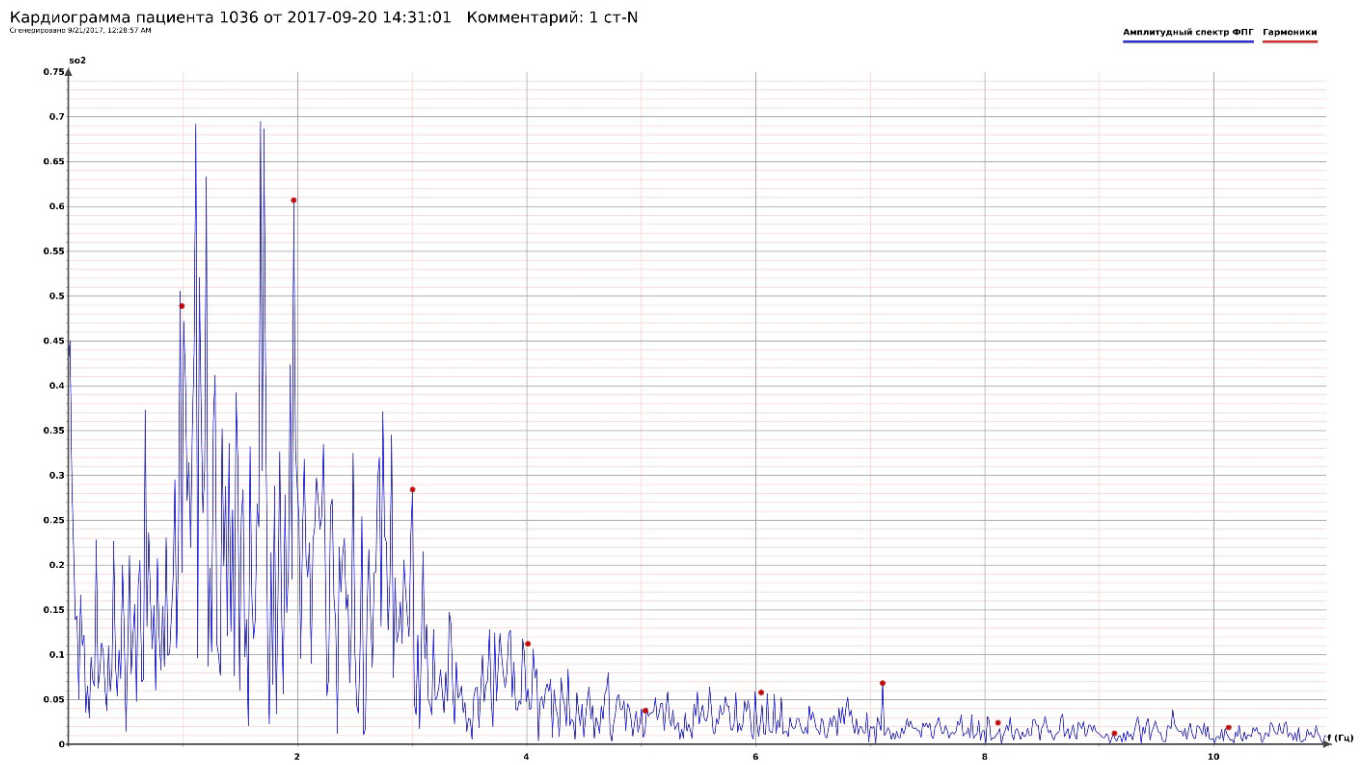

Figure 10: Real Fourier Pulse Wave image of a patient with a cardiac problem. 
Citation: Shmid AV, Novopashin MA, Berezin AA (2017) FPU Recurrence in the Coupled Blood Pressure Dynamics in Magisterial and Small Arteries Networks and the Character of its Interaction with the Heart Electrical Activity. Cardiovasc Pharm Open Access 6: 230. doi: 10.4172/23296607.1000230

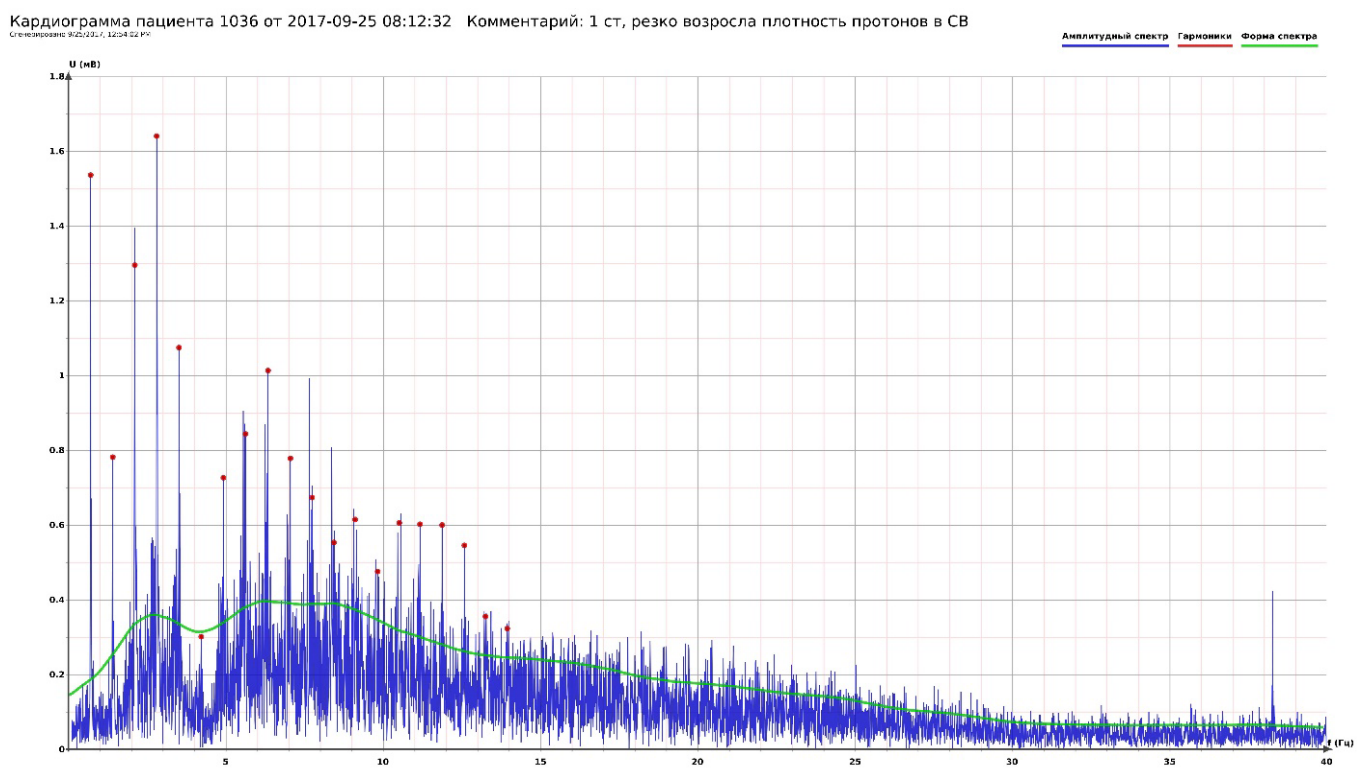

Figure 11: Improved Real Fourier ECG image of a patient with a cardiac problem after a course of therapy.

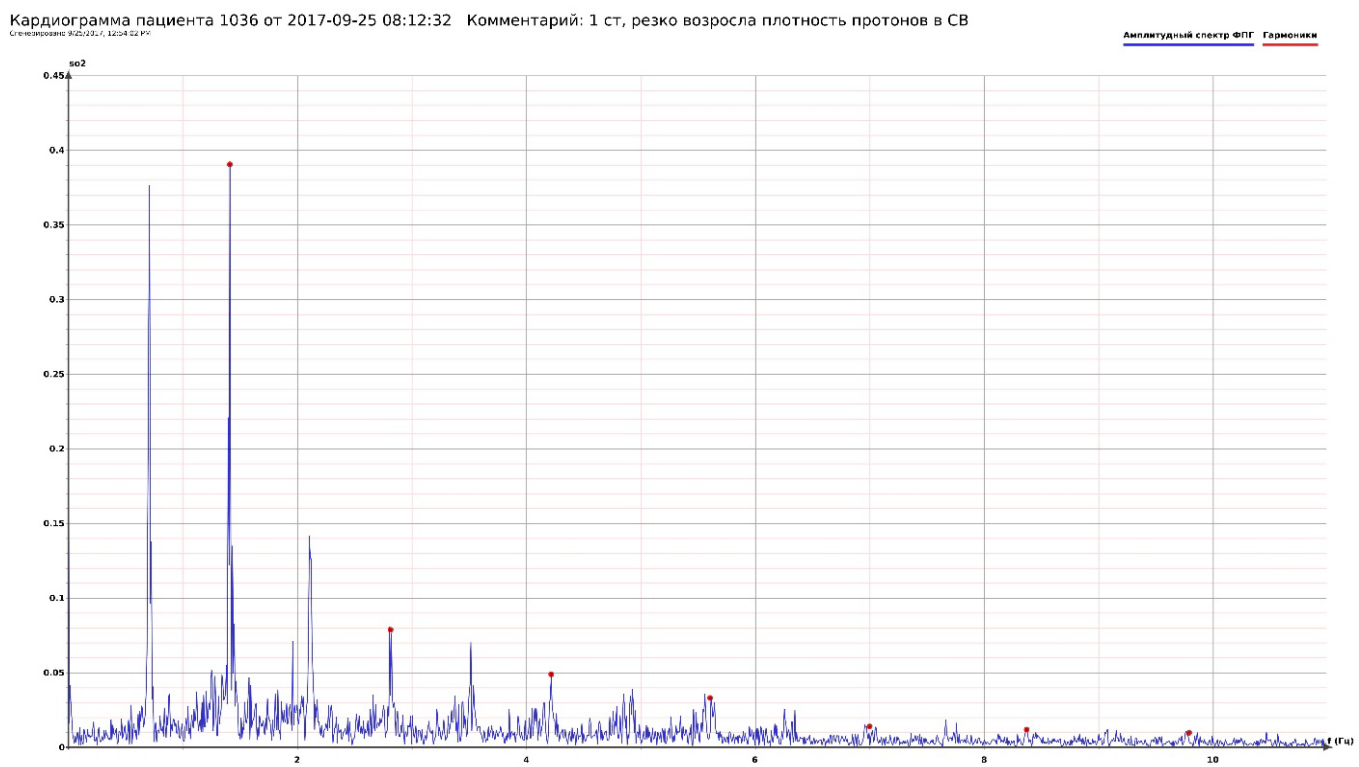

Figure 12: Improved Real Fourier Pulse Wave image of a patient with a cardiac problem after a course of therapy.

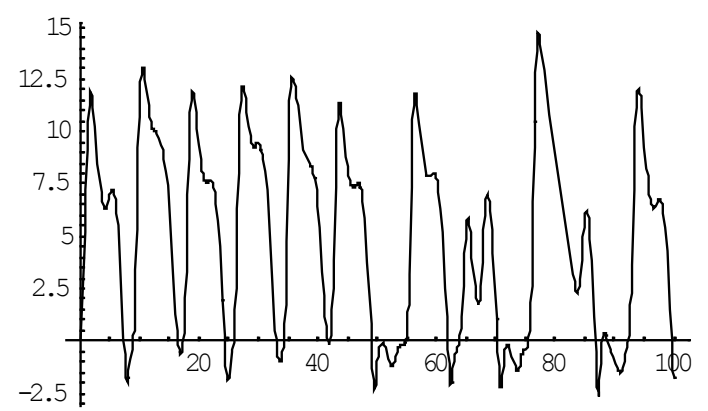

Figure 13: Model heart fibrillation partly stabilized by both arterial networks dynamics.

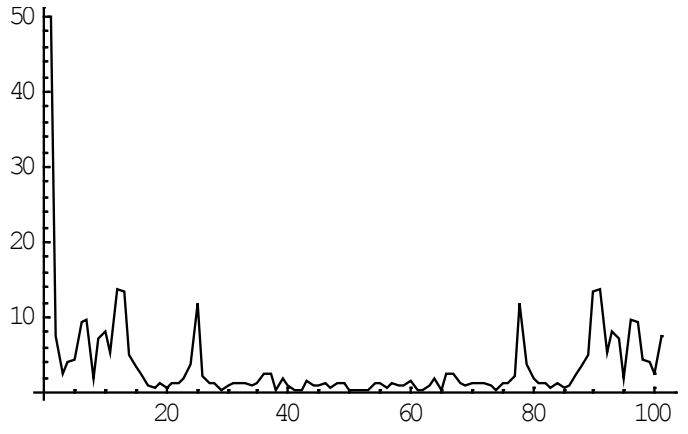

Figure 14: Model Fourier image of heart fibrillation partly stabilized by both arterial networks dynamics. 
Citation: Shmid AV, Novopashin MA, Berezin AA (2017) FPU Recurrence in the Coupled Blood Pressure Dynamics in Magisterial and Small Arteries Networks and the Character of its Interaction with the Heart Electrical Activity. Cardiovasc Pharm Open Access 6: 230. doi: 10.4172/23296607.1000230

Page 6 of 6

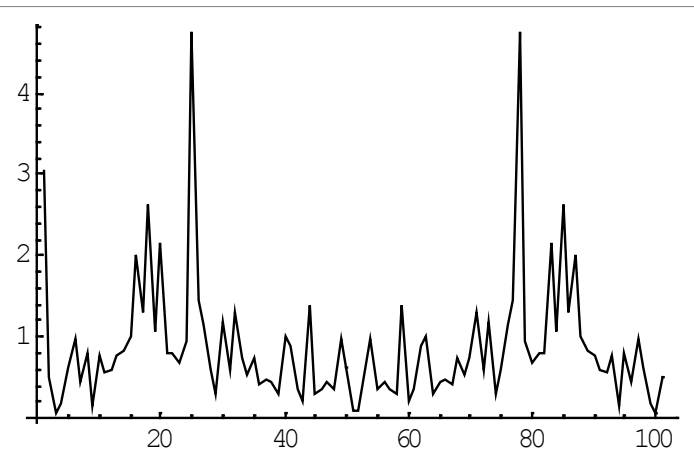

Figure 15: Model Fourier Pulse Wave image of the heart fibrillation partly stabilized by both arterial networks dynamics.

\section{Discussion}

The new mathematical model of the coupled system of the heart and magisterial and small arteries networks mutual functioning demonstrated the unified stable working of the system and its increased ability to resist cardiovascular problems such as myocardial infarction. The modeling dynamics of blood pressure in small arteries shows its ability to compensate the drop of blood pressure in magisterial arteries as well as its buffering property in cases of emotional stress. At the same time, the self-parametric excitation in the equations modeling the dynamics of blood pressure in small arteries points at their principal possibility of rupture or blowing up of healthy small arteries in some anomalous cases causing a brain hemorrhage or a heart attack. Those ones must be prevented in advance by therapeutic measures.

\section{References}

1. Novopashin MA, Shmid AV, Berezin AA (2017) Fermi-Pasta-Ulam auto recurrence in the description of the electrical activity of the heart. $\mathrm{J}$ Medical Hypothesis.

2. Yuen HC, Lake BM (1982) Non-linear dynamics of deep-water gravity waves. Advances in applied mechanics. Chia-Shun Yih 67: p 226.

3. Zabusky N, Kruskal M (1965) Interaction of solitons in a collision less plasma and recurrence of initial states. Physical Review Letters.

4. Novopashin MA, Shmid AV, Berezin AA (2017) Forrester's Concept in Modeling Heart Dynamics. IOSR J Computer Eng 19. 